Article

\title{
Triterpenoids and Steroids from Ganoderma mastoporum and Their Inhibitory Effects on Superoxide Anion Generation and Elastase Release
}

\section{Tran Dinh Thang ${ }^{1, \dagger}$, Ping-Chung Kuo ${ }^{2, \dagger}$, Tsong-Long Hwang ${ }^{3}$, Mei-Lin Yang ${ }^{4}$,} Nguyen Thi Bich Ngoc ${ }^{1}$, Tran Thi Ngoc Han ${ }^{1}$, Chi-Wen Lin ${ }^{2}$ and Tian-Shung Wu ${ }^{4, *}$

1 Department of Chemistry, Vinh University, Vinh City, Nghe An 42000, Vietnam; E-Mails: thangtd@vinhuni.edu.vn (T.D.T.); ngockhoahoa@gmail.com (N.T.B.N.); ngochan.tran1990@gmail.com (T.T.N.H.)

2 Department of Biotechnology, National Formosa University, Yunlin 632, Taiwan; E-Mails: pcckuoo@nfu.edu.tw (P.-C.K.); sai_free@yahoo.com.tw (C.-W.L.)

3 Graduate Institute of Natural Products and Chinese Herbal Medicine Research Team, Healthy Aging Research Center, Chang Gung University, Taoyuan 333, Taiwan; E-Mail: ht1@mail.cgu.edu.tw

4 Department of Chemistry, National Cheng Kung University, Tainan 701, Taiwan; E-Mail: L3891104@nckualumni.org.tw

$\dagger$ These authors contributed equally to this work.

* Author to whom correspondence should be addressed; E-Mail: tswu@mail.ncku.edu.tw; Tel: +886-6-275-7575 (ext. 65333); Fax: +886-6-274-0552.

Received: 27 September 2013; in revised form: 6 November 2013 / Accepted: 15 November 2013 / Published: 19 November 2013

\begin{abstract}
The methanol extracts of the fruiting bodies of Ganoderma mastoporum collected in Vietnam was purified to afford eight compounds, including three triterpenoids and five steroids. The purified compounds were examined for their inhibitory effects against superoxide anion generation and elastase release. Among the tested compounds, ergosta-4,6,8(14),22-tetraen-3-one (3) exhibited the most significant inhibition towards superoxide anion generation and elastase release with $\mathrm{IC}_{50}$ values of $2.30 \pm 0.38$ and $1.94 \pm 0.50 \mu \mathrm{g} / \mathrm{mL}$, respectively.
\end{abstract}

Keywords: Ganoderma; triterpenoid; steroid; superoxide anion generation; elastase release 


\section{Introduction}

The genus Ganoderma (Polyporaceae) are extensively used as a Chinese remedy for the treatment of tumors [1,2], hepatopathy [3], enhancement of splenic natural killer cell activity, and serum interferon production in mice [4]. Species such as G. lucidum attract much attention because it is a well-known source of various therapeutic agents [5,6]. Previous phytochemical investigations of Ganoderma genus afforded a series of triterpenoids and steroids [7-13]. These principles displayed significant cytotoxicity and anti-inflammatory bioactivity [14,15]. G. mastoporum is a fungus widely distributed in Southern China, Northern Vietnam, Malaysia, Philippines, and reported to contain sesquiterpenoids [16]. Therefore, additional studies of the phytochemical constituents of G. mastoporum and their bioactivities are merited. In our continuous program aimed at the discovery of anti-inflammatory lead drugs from the natural sources, the chemical composition of the fruiting bodies of G. mastoporum collected in Vietnam was investigated to search for the bioactive constituents. In the present study, we wished to report the identification of eight compounds, as well as their inhibitory effects on superoxide anion generation and elastase release.

\section{Results and Discussion}

\subsection{Isolation and Identification of Compounds $\mathbf{1}-\mathbf{8}$}

Air-dried and powdered fruiting bodies of Ganoderma mastoporum were extracted with methanol, and the combined extracts were concentrated under reduced pressure to give a deep brown syrup. The crude extract was suspended in water and partitioned with ethyl acetate to afford ethyl acetate and water soluble fractions, respectively. Purification of the ethyl acetate fraction by a conventional combination of column chromatographies yielded three triterpenes: $\Delta^{1}$-lupenone (1) [17], ganodermanondiol (5), lucidumol B (6) [18], and five steroids: ergosta-7,22-dien-3 $\beta$-ol (2) [19], ergosta-4,6,8(14),22-tetraen-3-one (3), ergosterol peroxide (4) [20], ergosta-7,22-dien-3-one (7) [19], and 3 $\beta, 5 \alpha$-dihydroxy-(22E,24R)-ergosta-7,22-dien-6-one (8) [21], respectively. Their chemical structures (Figure 1) were identified by comparison of their physical and spectroscopic data with those reported in the literature.

\subsection{The Inhibitory Effects of Isolated Compounds on Superoxide Anion Generation and Elastase Release}

The purified compounds were subjected to the examination for inhibition of superoxide anion generation and elastase release by human neutrophils in response to $\mathrm{N}$-formyl-L-methionyl-leucylphenylalanine/cytochalasin B (FMLP/CB) [22], and the data are displayed in Table 1. Only compounds $\mathbf{2}$ and $\mathbf{8}$ did not display significant inhibition of superoxide anion generation and elastase release. Other tested compounds $\mathbf{1}$ and 3-7 demonstrated inhibitory effects towards superoxide anion generation and elastase release in a concentration-dependent manner (data not shown). The $\mathrm{IC}_{50}$ values were in the range between $2.30 \pm 0.38$ and $6.36 \pm 0.36 \mu \mathrm{g} / \mathrm{mL}$ against superoxide anion generation, and between $1.94 \pm 0.50$ and $5.01 \pm 0.82 \mu \mathrm{g} / \mathrm{mL}$ against elastase release, respectively, compared with the reference compound LY294002 ( IC $_{50}$ of $0.40 \pm 0.02$ and $1.53 \pm 0.25 \mu \mathrm{g} / \mathrm{mL}$ towards superoxide anion generation and elastase release). Among the tested compounds, ergosta-4,6,8(14),22-tetraen-3-one (3) 
exhibited the most significant inhibition towards superoxide anion generation and elastase release with $\mathrm{IC}_{50}$ of $2.30 \pm 0.38$ and $1.94 \pm 0.50 \mu \mathrm{g} / \mathrm{mL}$. From the above results, the following structure-bioactivity relationships were deduced: triterpenoids or steroids possessing 3-hydroxy rather than 3-ketone functionalities, such as $\mathbf{2 , 4 , 6}$, and $\mathbf{8}$, displayed weaker superoxide anion generation inhibitory effects. Comparison of the bioactivity of $\mathbf{2}$ and $\mathbf{7}$ also exhibited this tendency. The 24,25-dihydroxy substitutions in $\mathbf{5}$ and $\mathbf{6}$, and the 5-hydroxy group in $\mathbf{8}$ decreased the inhibition of superoxide anion generation. There is similar tendency in the inhibition of elastase release. However, lucidumol B (6) displayed more significant inhibition of elastase release than $\mathbf{5}$ and $\mathbf{7 .}$

Figure 1. Structures of purified compounds 1-8.
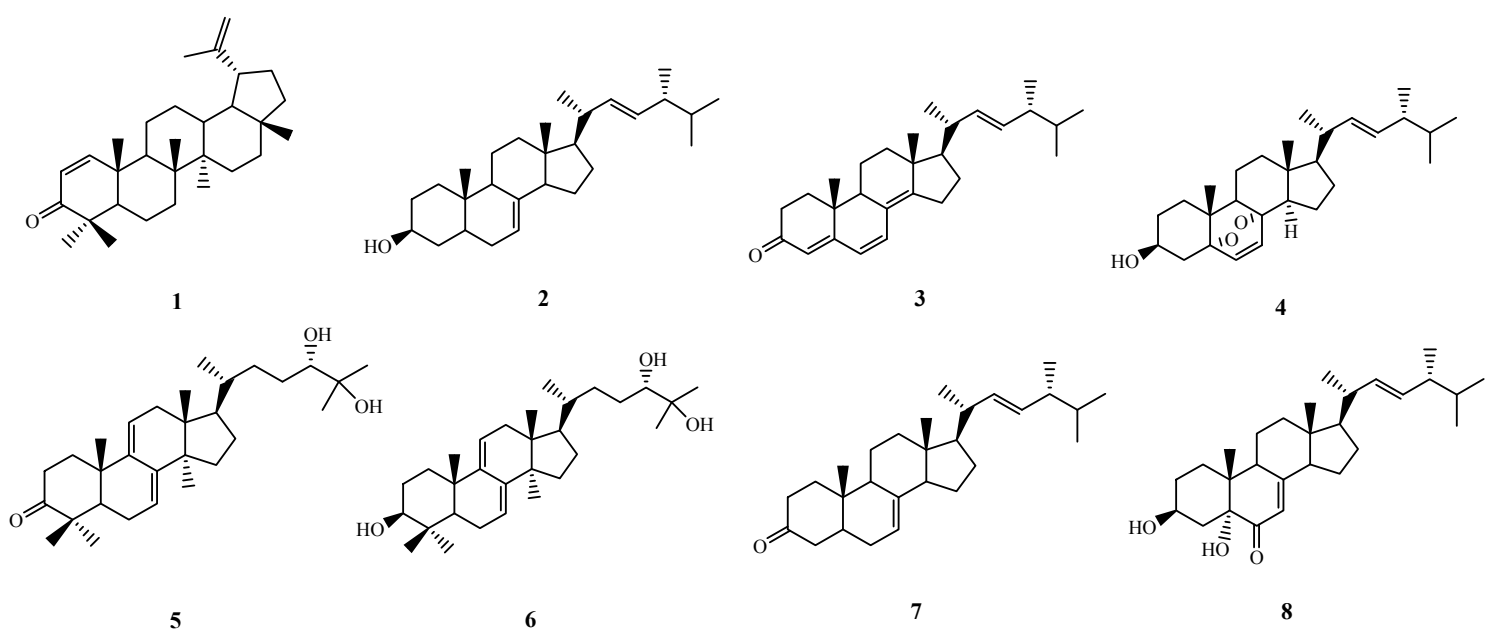

Table 1. Inhibitory effects of purified samples from G. mastoporum on superoxide anion generation and elastase release by human neutrophils in response to $N$-formyl-L-methionylleucyl-phenylalanine/cytochalasin B (FMLP/CB).

\begin{tabular}{ccc}
\hline \multirow{2}{*}{ Compound } & \multicolumn{2}{c}{$\mathbf{I C}_{\mathbf{5 0}}(\boldsymbol{\mu g} \mathbf{g} \mathbf{m L})^{\mathbf{a}}$ or $(\mathbf{I n h} \mathbf{\%}) \mathbf{b}^{\mathbf{b}}$} \\
\cline { 2 - 3 } & Superoxide Anion Generation & Elastase Release \\
\hline $\mathbf{1}$ & $3.71 \pm 0.79^{* * *}$ & $3.26 \pm 0.07 * * *$ \\
$\mathbf{2}$ & $(34.46 \pm 2.42)^{* * *}$ & $(27.44 \pm 4.90) * *$ \\
$\mathbf{3}$ & $2.30 \pm 0.38^{* * *}$ & $1.94 \pm 0.50 * * *$ \\
$\mathbf{4}$ & $5.28 \pm 0.76^{* * *}$ & $(35.99 \pm 3.42)^{* * *}$ \\
$\mathbf{5}$ & $6.36 \pm 0.36^{* * *}$ & $5.01 \pm 0.82^{* *}$ \\
$\mathbf{6}$ & $5.33 \pm 0.46^{* * *}$ & $3.32 \pm 0.14 * * *$ \\
$\mathbf{7}$ & $5.02 \pm 0.98^{* * *}$ & $4.41 \pm 0.50 * * *$ \\
$\mathbf{8}$ & $(30.11 \pm 3.17)^{* * *}$ & $(19.46 \pm 6.65)^{*}$ \\
$\mathbf{L Y 2 9 4 0 0 2}^{\mathrm{c}}$ & $0.40 \pm 0.02^{* * *}$ & $1.53 \pm 0.25 * * *$ \\
\hline
\end{tabular}

${ }^{\mathrm{a}}$ Concentration necessary for $50 \%$ inhibition. ${ }^{\mathrm{b}}$ Percentage of inhibition (Inh \%) at $10 \mu \mathrm{g} / \mathrm{mL}$ concentration; results are presented as mean \pm S.E.M. $(\mathrm{n}=3-4) ;{ }^{*} p<0.05 ; * * p<0.01 ; * * * p<0.001$ compared with the control value. ${ }^{\mathrm{c}}$ A phosphatidylinositol-3-kinase inhibitor was used as a positive control for superoxide anion generation and elastase release.

The formation of superoxide anion in neutrophils can be inhibited by modulating cellular signaling pathways, but also by direct radical scavenging. Therefore the purified constituents $\mathbf{1}-\mathbf{8}$ were 
also subjected to assays of DPPH free radical scavenging activities and ferrous ion chelating capability [23]. None of the examined compounds exhibited significant scavenging of free radicals and capability of chelating ferrous ions at the tested concentration (data not shown). It indicated that the inhibition of superoxide anion generation and elastase release by compounds $\mathbf{1}$ and $\mathbf{3}-\mathbf{7}$ is mediated by modulating cellular signaling pathways [24]. In the previous literature, $\Delta^{1}$-lupenone (1) and ergosterol peroxide (4) were reported to exhibit the inhibitory effects on LPS-induced $i$ NOS-dependent NO production in RAW 264.7 cells [25-27]. Moreover, ergosta-7,22-dien-3 $\beta$-ol (2), ergosterol peroxide (4), and ergosta-7,22-dien-3-one (7) also inhibited superoxide anion generation release by rat neutrophils [15]. In the present study, the inhibitory effects of these isolated compounds on superoxide anion generation and elastase release by human neutrophils in response to FMLP/CB were reported for the first time.

\section{Experimental}

\subsection{General Procedure}

Melting points were determined using Yanagimoto MP-S3 apparatus (Kyoto, Japan). Optical rotations were measured using a JASCO DIP-370 polarimeter (Oklahoma City, OK, USA). The UV spectra were obtained on a Hitachi UV-3210 spectrophotometer (Tokyo, Japan), and IR spectra were recorded on a Shimadzu FTIR-8501 spectrophotometer (Kyoto, Japan). ${ }^{1} \mathrm{H}$ - and ${ }^{13} \mathrm{C}-\mathrm{NMR}$, COSY, NOESY, HMQC, and HMBC spectra were obtained on the Bruker Avance III-400 NMR spectrometer (Fremont, CA, USA), with tetramethylsilane (TMS) as internal standard and the chemical shifts were reported in $\delta$ values (ppm). The electrospray ionization (ESI) mass spectrum was determined using an Agilent 1200 LC-MSD Trap spectrometer (Santa Clara, CA, USA). Column chromatography (CC) was performed on silica gel (Kieselgel 60, 70-230 mesh and 230-400 mesh, E. Merck, Darmstadt, Germany). Thin layer chromatography (TLC) was conducted on precoated Kieselgel $60 \mathrm{~F} 254$ plates (Merck) and the compounds were visualized by spraying with $10 \%(\mathrm{v} / \mathrm{v}) \mathrm{H}_{2} \mathrm{SO}_{4}$ followed by heating at $110^{\circ} \mathrm{C}$ for $10 \mathrm{~min}$.

\subsection{Fungus Materials}

The basidiomycete (Ganoderma mastoporum (Mont) Pat.) was collected at the Puhuong National Park of Nghean Province, Vietnam, in November 2010 and identified by Dr. Ngo Anh, Department of Biology, Hue University. A voucher specimen (Vinh-TSWu 20100805) was deposited at the herbarium of the Department of Chemistry, Vinh University.

\subsection{Extraction and Isolation}

The fruiting bodies of Ganoderma mastoporum $(4.3 \mathrm{Kg})$ were air-dried, powdered, and extracted with methanol $(10 \mathrm{~L} \times 3)$ at ambient temperature, and the combined extracts were concentrated under reduced pressure to give a deep brown syrup (125 g). The crude extract was suspended in water and partitioned with ethyl acetate to afford ethyl acetate $(30 \mathrm{~g})$ and water soluble $(95 \mathrm{~g})$ fractions, respectively.

The ethyl acetate soluble extracts were subjected to silica gel column chromatography (CC) with $n$-hexane and acetone gradients (15:1 to $1: 1)$ to afford five fractions. Fractions $1-2$ and $4-5$ did not 
display significant triterpenoid and steroid spots monitored by TLC so that they were not further purified. Fraction 3 was a triterpene-rich fraction visualized by spraying with $10 \%(\mathrm{v} / \mathrm{v}) \mathrm{H}_{2} \mathrm{SO}_{4}$ followed by heating at $110{ }^{\circ} \mathrm{C}$ for $10 \mathrm{~min}$. It was subjected to silica gel column chromatography eluted with chloroform and methanol gradients (100:1 to 1:1) to afford three subfractions. Subfraction 3-1 was purified by $\mathrm{SiO}_{2} \mathrm{CC}$ (eluted with chloroform/ethyl acetate 20:1) and further recrystallization of the resulted minor fractions with chloroform/methanol to yield $\Delta^{1}$-lupenone $(1,4 \mathrm{mg})$ and ergosta-7,22-dien-3 $\beta$-ol (2, $6 \mathrm{mg})$, respectively. Subfraction 3-2 was repeatedly purified by $\mathrm{SiO}_{2} \mathrm{CC}$ and preparative TLC (pTLC) (eluted with benzene/ethyl acetate 20:1) to afford ergosta-4,6,8(14),22tetraen-3-one (3, $3 \mathrm{mg})$, ergosterol peroxide (4, $8 \mathrm{mg})$, and ganodermanondiol $(5,2 \mathrm{mg})$, respectively. Subfraction 3-3 was purified by $\mathrm{SiO}_{2} \mathrm{CC}$ (eluted with benzene/acetone 50:1) to yield three minor fractions (3-3-1 3-3-3). Fractions 3-3-1 and 3-3-2 were repeatedly purified by $\mathrm{SiO}_{2} \mathrm{CC}$ and pTLC (eluted with benzene/ethyl acetate 20:1) to afford lucidumol B (6, $3 \mathrm{mg}$ ), and ergosta-7,22-dien-3-one (7, $2 \mathrm{mg})$, respectively. Fractions 3-3-3 was further recrystallized with chloroform /methanol to result in 3 $\beta, 5 \alpha$-dihydroxy-(22E,24R)-ergosta-7,22-dien-6-one (8, $2 \mathrm{mg})$.

\subsection{Determination of Inhibitory Effects on Superoxide Anion Generation and Elastase Release}

\subsubsection{Preparation of Human Neutrophils}

Neutrophils were isolated with a standard method of dextran sedimentation prior to centrifugation in a Ficoll Hypaque gradient and hypotonic lysis of erythrocytes. Blood was drawn from healthy human donors (20-30 years old) by venipuncture into heparin-coated vacutainer tubes, using a protocol approved by the institutional review board at Chang Gung Memorial Hospital [22].

\subsubsection{Measurement of Superoxide Anion Generation}

The assay of the generation of superoxide anion was based on the SOD-inhibitable reduction of ferricytochrome c [22]. Calculations were based on differences in the reactions with and without SOD $(100 \mathrm{U} / \mathrm{mL})$ divided by the extinction coefficient for the reduction of ferricytochrome c $(\varepsilon=21.1 / \mathrm{mM} / 10 \mathrm{~mm})$.

\subsubsection{Measurement of Elastase Release}

Degranulation of azurophilic granules was determined by elastase release as described previously [22]. The results were expressed as the percent of elastase release in the FMLP/CB-activated, drug-free control system.

\subsubsection{Assay of Scavenging Activity against Diphenyl Picrylhydrazyl (DPPH) Radical}

The DPPH free radical scavenging assay was executed according to the reported method [23]. The DPPH radical scavenging activities of the sample solutions were expressed as inhibition percentage and calculated by using the following equation:

Inhibition percentage $(\%)=[1-($ absorbance of samples at $517 \mathrm{~nm}) /$

(absorbance of control at $517 \mathrm{~nm})] \times 100(\%)$. 


\subsubsection{Chelation of Ferrous Ions}

The capability of chelating ferrous ions was determined by the reported method with some modifications [23]. The relative capability of chelating ferrous ions was derived from the formula:

$$
[1-(\text { sample absorbance at } 562 \mathrm{~nm}) /(\text { control absorbance at } 562 \mathrm{~nm})] \times 100(\%)
$$

\subsubsection{Statistical Analysis}

Results were expressed as mean \pm S.E.M. Computation of $50 \%$ inhibitory concentration $\left(\mathrm{IC}_{50}\right)$ was computer-assisted (PHARM/PCS v.4.2). Statistical comparisons were made between groups using Student's $t$ test. Values of $p$ less than 0.05 were considered to be statistically significant.

\section{Conclusion}

In summary, three triterpenoids and five steroids from the fruiting bodies of G. mastoporum collected in Vietnam were characterized and their inhibition of superoxide anion generation and elastase release by human neutrophils in response to FMLP/cytochalasin B was examined. The results provide evidence for the use of the fruiting bodies of $G$. mastoporum as herbal medicines in the treatment of inflammatory diseases, and these purified principles may be potentially useful in developing new anti-inflammatory therapeutic agents.

\section{Acknowledgments}

The authors are thankful to the National Science Council, Taiwan, for financial support of the present research. This study was supported in part by MOST (15/2012/HĐ-NĐT) (Vietnam).

\section{Conflicts of Interest}

The authors declare no conflict of interest.

\section{References}

1. Ruan, Z.; Su, J.; Dai, H.C.; Wu, M.C. Characterisation and immunomodulating activities of polysaccharides from Lentinus edodes. Int. Immunopharmacol. 2005, 5, 811-820.

2. Stanley, G.; Harvey, K.; Slivova, V.; Jiang, J.; Sliva, D. Ganoderma lucidum suppresses angiogenesis through the inhibition of secretion of VEGF and TGF-B1 from prostate cancer cells. Biochem. Biophys. Res. Commun. 2005, 330, 46-52.

3. Aarisawa, M.; Fujita, A.; Saga, M.; Fukumura, H.; Hayashi, T.; Shimizu, M.; Morita, N. Three new lanostanoids from Ganoderma lucidum. J. Nat. Prod. 1986, 49, 621-625.

4. Won, S.J.; Lin, M.T.; Wu, W.L. Ganoderma tsugae mycelium enhances splenic natural killer cell activity and serum interferon production in mice. Japan J. Pharmacol. 1992, 59, 171-176.

5. Paterson, R.R.M. Ganoderma-A therapeutic fungal biofactory. Phytochemistry 2004, 67, 1985-2001. 
6. Wasser, S.P. Medicinal mushrooms as a source of antitumor and immunomodulating polysaccharides. Appl. Microbiol. Biotechnol. 2002, 60, 258-274.

7. Lin, L.J.; Shiao, M.S. ${ }^{13}$ C-NMR correlation of stereochemistry in lanostanoids triterpenes. J. Nat. Prod. 1989, 52, 595-605.

8. Lin, C.N.; Tome, W.P.; Won, S.J. A lanostanoid of Formosan Ganoderma lucidum. Phytochemistry 1990, 29, 673-675.

9. Lin, C.N.; Kuo, S.H.; Won, S.J. Steroids of Formosan Ganoderma amboinense. Phytochemistry 1993, 32, 1549-1551.

10. Lin, C.N.; Fann, Y.F.; Chung, M.I. Steroids of Formosan Ganoderma tsugae. Phytochemistry 1997, 46, 1143-1146.

11. Gan, K.H.; Fann, Y.F.; Hsu, S.H.; Kuo, K.W.; Lin, C.N. Mediation of cytotoxicity of lanostanoids and steroids of Ganoderma tsugae through apoptosis and cell cycle. J. Nat. Prod. 1998, 61, 485-487.

12. Gan, K.H.; Kuo, S.S.; Lin, C.N. Steroidal constituents of Ganoderma applanatum and Ganoderma neo-japonicum. J. Nat. Prod. 1998, 61, 1421-1422.

13. Su, H.J.; Fann, Y.F.; Chung, M.I.; Won, S.J.; Lin, C.N. New lanostanoids of Ganoderma tsugae. J. Nat. Prod. 2000, 63, 514-516.

14. Giner-Larza, E.M.; Máñez, S.; Giner-Pons, R.M.; Recio, M.C.; Ríos, J. On the anti-inflammatory and anti-phospholipase A2 activity of extracts from lanostane-rich species. J. Ethnopharmacol. 2000, 73, 61-69.

15. Ko, H.H.; Hung, C.F.; Wang, J.P.; Lin, C.N. Antiinflammatory triterpenoids and steroids from Ganoderma lucidum and G. tsugae. Phytochemistry 2008, 69, 234-239.

16. Hirotani, M.; Ino, C.; Hatano, A.; Takayanagi, H.; Furuya, T. Ganomastenols A, B, C and D, cadinene sesquiterpenes from Ganoderma mastoporum. Phytochemistry 1995, 40, 161-165.

17. Srivastava, R.; Kulshreshtha, D.K. Triterpenoids from Glochidion heyneanum. Phytochemistry 1988, 27, 3575-3578.

18. Fujita, A.; Arisawa, M.; Saga, M.; Hayashi, T.; Morita, N. Two new lanostanoids from Ganoderma lucidum. J. Nat. Prod. 1986, 49, 1122-1125.

19. Lu, W.; Adachi, I.; Kano, K.; Yasuta, A.; Toriizuka, K.; Ueno, M.; Horikoshi, I. Platelet aggregation potentiators from Cho-Rei. Chem. Pharm. Bull. 1985, 33, 5083-5087.

20. Jinming, G.; Lin, H.; Jikai, L. A novel sterol from chinese truffles Tuber Indicum. Steroids 2001, $66,771-775$.

21. Ishizuka, T.; Yaoita, Y.; Kikuchi, M. Sterol constituents from the fruit bodies of Grifola frondosa (Fr.) S. F. Gray. Chem. Pharm. Bull. 1997, 45, 1756-1760.

22. Hwang, T.L.; Li, G.L.; Lan, Y.H.; Chia, Y.C.; Hsieh, P.W.; Wu, Y.H.; Wu, Y.C. Potent inhibitors of superoxide anion production in activated human neutrophils by isopedicin, a bioactive component of the Chinese medicinal herb Fissistigma oldhamii. Free Radic. Biol. Med. 2009, 46, $520-528$.

23. Kuo, P.C.; Lin, M.C.; Chen, G.F.; Yiu, T.J.; Tzen, J.T.C. Identification of methanol-soluble compounds in sesame and evaluation of antioxidant potential of its lignans. J. Agric. Food Chem. 2011, 59, 3214-3219. 
24. Yang, M.L.; Kuo, P.C.; Hwang, T.L.; Chiou, W.F.; Qian, K.; Lai, C.Y.; Lee, K.H.; Wu, T.S. Synthesis, in vitro anti-inflammatory and cytotoxic evaluation, and mechanism of action studies of 1-benzoyl- $\beta$-carboline and 1-benzoyl-3-carboxy- $\beta$-carboline derivatives. Bioorg. Med. Chem. 2011, 19, 1674-1682.

25. Jin, S.E.; Son, Y.K.; Min, B.S.; Jung, H.A.; Choi, J.S. Anti-inflammatory and antioxidant activities of constituents isolated from Pueraria lobata roots. Arch. Pharm. Res. 2012, 35, 823-837.

26. Chao, W.W.; Kuo, Y.H.; Lin, B.F. Anti-inflammatory activity of new compounds from Andrographis paniculata by NF-кB transactivation inhibition. J. Agric. Food Chem. 2010, 58, 2505-2512.

27. Ma, L.; Chen, H.; Dong, P.; Lu, X. Anti-inflammatory and anticancer activities of extracts and compounds from the mushroom Inonotus obliquus. Food Chem. 2013, 139, 503-508.

Sample Availability: Samples of the compounds 1-8 are available from the authors.

(C) 2013 by the authors; licensee MDPI, Basel, Switzerland. This article is an open access article distributed under the terms and conditions of the Creative Commons Attribution license (http://creativecommons.org/licenses/by/3.0/). 